\title{
Consideraciones Teóricas Acerca del Empoderamiento Digital y Desarrollo Sostenible del Sector Empresarial en Colombia
}

\author{
Theoretical Considerations about Digital Empowerment and Sustainable \\ Development of the Business Sector in Colombia
}

Iris A. Jiménez-Pitre ${ }^{1}$, Geomar Molina-Bolivar ${ }^{2}$ y Rodrigo Gámez-Pitre ${ }^{3}$

\section{EDICIÓN. Resiliencia}

Recibido: 16/julio/2021

Aceptado: 17/octubre/2021

จ Publicado: 29/noviembre/2021

Páginas: 163-173

\&aís

${ }^{1}$ Colombia

${ }^{2}$ Colombia

${ }^{3}$ Colombia

\section{IIIIInstitución}

${ }^{1}$ Universidad de La Guajira

${ }^{2}$ Universidad de La Guajira

${ }^{3}$ Universidad de La Guajira

\section{Correo Eletrónico}

1iajimenez@uniguajira.edu.co ²gmolina@uniguajira.edu.co 3rgamez@uniguajira.edu.co

\section{(D) ORCID}

${ }^{1}$ https://orcid.org/0000-0002-8109-7013 ${ }^{2} \mathrm{https} / / /$ orcid.org/0000-0002-3119-9265 ${ }^{3} \mathrm{https}: / /$ orcid.org/0000-0003-1436-097

\section{Citar así: CfAPA / IEEE}

Jiménez-Pitre, I., Molina-Bolivar, G. \& Gámez-Pitre, R. (2021). Consideraciones Teóricas Acerca del Empoderamiento Digital y Desarrollo Sostenible del Sector Empresarial en Colombia. Revista Tecnológica-Educativa Docentes 2.0, 1(1), 163-173.

https://doi.org/10.37843/rted.v1i1.267

I. Jiménez-Pitre, G. Molina-Bolivar y R. Gámez-Pitre, "Consideraciones Teóricas Acerca del Empoderamiento Digital y Desarrollo Sostenible del Sector Empresarial en Colombia", RTED, vol. 1, n. ${ }^{\circ} 1$, pp. 163-173, nov. 2021.

\section{Resumen}

El auge que han alcanzado las tecnologías está influenciando todo el panorama del quehacer productivo y social de las personas. Todo esto desde una perspectiva de desarrollo económico y científico, promoviendo una gama de oportunidades para transformar el dinamismo social y productivo, en otras palabras, desde una concepción económica. De allí, que este estudio se fijó como objetivos establecer una concepción teórica acerca del empoderamiento digital y el desarrollo sostenible del sector empresarial. Asimismo, se orientó a determinar la relación entre el empoderamiento digital y desarrollo sostenible del sector empresarial en Colombia. Por lo tanto, se afianzó en una metodología positivista, tipificada como analítica - descriptiva y correlacional, la muestra estuvo conformada por 32 docentes universitarios y 22 estudiantes que han incursionado en el sector laboral. La información se recolectó mediante un cuestionario estructurado, con alternativas de respuestas múltiples. Los resultados mostraron respecto al primer objetivo que la concepción que se tiene sobre el empoderamiento digital es endeble, asumido como el uso de las TIC. Respecto al segundo objetivo se evidenció una correlación de 0,763 a un nivel de significancia de 0,033 , indicando con esto que hay una relación alta y estadísticamente significativa entre las variables.

Palabras clave: Empoderamiento digital, empresas, predictores de la integración, desarrollo sostenible.

\section{Abstract}

The upswing that technologies have reached is influencing the entire panorama of people's productivity and social work. All this from an economic and scientific development perspective, promoting a range of opportunities to transform social and productive dynamism, in other words, from an economic conception. Hence, this study's objectives were to establish a theoretical conception about digital empowerment and the sustainable development of the business sector. Likewise, it was oriented to determine the relationship between digital empowerment and the sustainable development of Colombia's business sector. Therefore, it was established in a positivist methodology, typified as analytical descriptive, and correlational; the sample consisted of 32 university teachers and 22 students who have ventured into the labor sector. The information was collected through a structured questionnaire with multiple response alternatives. The results showed regarding the first objective that the conception of digital empowerment is weak, assumed as the use of ICT. Regarding the second objective, a correlation of 0.763 was evidenced at a significance level of 0.033 , indicating a high and statistically significant relationship between the variables.

Keywords: Digital empowerment, companies, integration, sustainable development. 


\section{Introducción}

El auge que han alcanzado las tecnologías está influenciando todo el panorama del quehacer productivo y social de las personas. Todo esto desde una perspectiva de desarrollo económico y científico, promoviendo una gama de oportunidades para transformar el dinamismo social y productivo, en otras palabras, desde una concepción económica. De allí que las organizaciones requieren orientar los procesos en función del avance tecnológico. En este sentido, se amerita la reestructuración de los procedimientos y métodos en función de los nuevos escenarios, lo que quiere decir que toda empresa está llamada a implementar y aprovechar los recursos tecnológicos a partir del empoderamiento digital.

Dentro de este contexto, Carruyo, Ureña \& Quiñones (2017) señalan que el empoderamiento digital a nivel empresarial y en relación con la gestión de políticas públicas, se ocupa de brindar alternativas basadas en el uso de técnicas, estrategias y metodologías colaborativas, donde sean más eficientes los procesos y canales de comunicación, sin las limitantes de espacio y tiempo. Además, el uso de las tecnologías facilita el acceso a la información lo que repercute significativamente en el fundamento para la construcción del conocimiento. Asimismo, Guitert (2013) considera que otro elemento que debe considerarse a nivel empresarial es la formación del personal para que hagan uso eficiente de las mismas (tecnologías), además, cuando se menciona su correcto uso se alude el acceso, gestión y proceso la información, de manera que sea presentada en el formato adecuado.

Ahora bien, el empoderamiento digital al estar relacionado con capacidades y desarrollo de habilidades alude una forma de capacitación, para profesionales y futuros profesionales, lo que conlleva a pensar que, desde las instituciones educativas, específicamente las universidades se promueva el uso de las tecnologías, como parte del desarrollo sostenible de las naciones. Formando al estudiante de manera que puedan hacer frente a los retos tecnológicos y sociales, no solo como agentes con conocimientos en áreas específicas, (médicos, abogados, docentes, ingenieros, entre otros) sino que estos conocimientos converjan con habilidades tecnológicas para que puedan ir cónsonamente desenvolviéndose eficientemente en su contexto de acción profesional y laboral, yendo de la mano con las herramientas que los mercados internacionales ofrecen y las sociedades imponen.

Al respecto, Jiménez-Pitre et al. (2017), exponen que, las tecnologías actualmente han logrado un dominio casi absoluto. Indicándose de esta manera, que el empoderamiento digital forma parte del desarrollo sostenible del sector empresarial. De allí que en Colombia las tecnologías adquieren una nueva idea que involucra el redimensionamiento de los procesos tácitos en desarrollo económico y científico de la nación. Sin embargo, existe un vacío en cuanto a las concepciones teóricas implícitas en el empoderamiento digital para el desarrollo sostenible. Situación que direcciona los objetivos de este estudio, ya que se pretende establecer una concepción teórica acerca del empoderamiento digital y desarrollo sostenible del sector empresarial y determinar la relación entre el empoderamiento digital y desarrollo sostenible del sector empresarial en Colombia.

\section{Metodología}

Considerando los objetivos del estudio, se asumió una combinación de métodos de investigación, principalmente contempla los procedimientos de la investigación analítica descriptiva; la primera definida por GarzaMercado (2007) este tipo de investigación dirige los procedimientos a descubrir los elementos que hacen parte de un fenómeno de estudio en su totalidad y las interconexiones que da cuenta de su integración. Asimismo, 
plantea que el análisis como procesamiento de información cualitativa y datos estadísticos se realiza en las investigaciones descriptivas, sin embargo, dependen del nivel de investigación con el cual el investigador concluye.

Por otra parte, atendiendo a los objetivos del estudio, el cual pretendió determinar la relación entre el empoderamiento digital y desarrollo sostenible del sector empresarial en Colombia, se estableció una metodología correlacional definida por Tamayo y Tamayo (2009), como aquella que busca, determinar el grado en el cual las variables en uno o varios factores son concomitantes con la variación de uno $\mathrm{u}$ otros factores. Por ello, en esta investigación se identifican, describen y definen las características, propiedades y conductas de las variables empoderamiento digital y desarrollo sostenible del sector empresarial.

Asimismo, autores como Méndez \& Austrillo (2008), considera que, en las investigaciones correlaciónales se examinan las relaciones entre las variables o sus resultados, pero sin explicar que una sea causa de la otra. Bien sea, la importancia radica en este tipo de investigaciones en medir el nivel de relación entre ambas. Al final de la investigación se estableció su nivel de correlación por medio de la aplicación de la formula Rho de Spearman. En cuanto a la técnica de recolección de información se elaboró un cuestionario con 12 ítem de alternativas de respuestas múltiples, aplicado a una población constituida por 32 docentes universitarios y 22 estudiantes trabajadores.

Una vez recopilados los datos mediante los instrumentos diseñados para este fin, fue necesario procesarlos, es decir, elaborarlos matemáticamente, ya que la cuantificación y su tratamiento estadístico permiten llegar a conclusiones. Según Tamayo y Tamayo (2008), cualquiera que sea la técnica empleada para ello, no es otra cosa que el registro de los datos obtenidos por los instrumentos empleados, mediante una técnica analítica en la cual se obtuvieron las conclusiones
Para el análisis de los datos aportados por el instrumento se utilizó la estadística descriptiva, a través de la cual se presentaron los datos en una matriz de doble entrada, donde se ubicaron en la parte superior los ítems agrupados por bloques en atención a los indicadores, dimensiones y la variable, en el lado izquierdo se anotaron los sujetos de la investigación esto permitió obtener tablas y gráficos, utilizando para ello el paquete estadístico SPSS versión 21.0. Se estableció el grado de correlación entre Ética del gerente educativo y Desempeño docente utilizando la fórmula de Rho de Spearman, Posteriormente, se realizó el contraste de estos resultados con las teorías que sustentan el estudio, reseñando las conclusiones a que dieron lugar, presentando las recomendaciones pertinentes a estos resultados. A continuación, se describe la fórmula de Rho Spearman.

$$
R H o=1-\frac{\sum_{i=1}^{n} D i^{2}}{n x\left(n^{2}-1\right)}
$$

Donde

Rho $=$ Coeficiente de correlación de Sperman

$\mathrm{Di}=$ Diferencia entre los rangos de $\mathrm{i}$

$\mathrm{N}=$ Tamaño de la muestra

$1=$ Constante

\section{Resultados}

\section{Empoderamiento Digital: Mecanismo para el Desarrollo Sostenible}

El empoderamiento digital implica ir más allá del implemento de las tecnologías, se requiere de ciertas habilidades para que el uso de las mismas tenga una intencionalidad fijada, mediante la cual se tracen los mecanismos para alcanzar un alto nivel de producción. Cuando se sugieren el término empoderamiento digital se hace referencia a trascender a las bondades y beneficios que las tecnologías ofrecen, por lo tanto, representan un sinónimo de innovación y dinamismo que simplifica los procedimientos, de esta manera, se invierte menos tiempo. A criterio de Rodríguez, Acuña, Rojas \& Lobato (2015), el término refiere a una forma de conducir a las 
empresas a optimizar la producción y los servicios. De allí quien que para su utilización se debe contar con destrezas y habilidades para intervenir en la construcción y producción de nuevos servicios, según los propios intereses $\mathrm{y}$ anhelos de las organizaciones y las personas.

Los autores enfatizan que el conocimiento sobre el manejo de las tecnologías sin que se tenga una participación activa es solo una forma de reconocer estas herramientas como un instrumento o insumo más de producción, de allí que cuando se pone de manifiesto esta capacidad tecnológica y se acciona en beneficio de una organización o dentro de las políticas públicas, universidades y empresas cobran mayor valor y es aquí donde adquiere la denominación de empoderamiento digital.

Dada la importancia y trascendencia de las teologías en el mundo actualmente se hace necesario su gestión para apoyar a las empresas minimizando los esfuerzos y organizando los procedimientos de manera que se agilicen y se produzca un mejor servicio. Hoy en día es apremiante que el manejo adecuado (empoderamiento) de las TIC forme parte de las estrategias operativas de toda institución, sin importar el ámbito de acción de las mismas, es decir, que estas (TIC) sirvan de mecanismo para el uso eficaz de los recursos, su medición y control. Lo que influye directamente en la calidad de los productos, ya que el uso adecuado de las tecnologías se convierte para las empresas en una ventaja competitiva, que canaliza mejores formas de ejercer y orientar el rumbo de las organizaciones, todo en busca de mejorar los servicios.

Según Mochi (2012), para que las empresas se empoderen digitalmente no basta con instalar programas sofisticados de computadoras, también se requiere la adquisición de técnicas y habilidades en el manejo del hardware y el software, además, desarrollar capacidades para afrontar las necesidades concretas de las personas en cada ámbito en el cual se desenvuelva para mejorar sus condiciones de vida, laborales, profesionales y sociales. Al respecto de los beneficios sociales estos se vinculan con el aspecto laboral, lo que quiere decir, que a medida que los individuos se desarrollan personalmente, logran mejorar también a nivel profesionalmente y esto se traduce en mayor productividad y desempeño laborar en las empresas.

Atendiendo a los objetivos de este estudio, particularmente al que pretende establecer una concepción teórica acerca del empoderamiento digital y desarrollo sostenible del sector empresarial se tiene que existen muchas interpretaciones teóricas sobre el empoderamiento, sin embargo, para Gargallo-Castel \& Pérez-Sanz (2009) existen estudios recientes que define el empoderamiento como el dominios que los usuarios de la internet y las tecnologías tienen sobre ellas, sacando beneficios de todas aquellas alternativas que ofrecen. Adaptando cada procedimiento y objetivo empresarial a un manejo más eficiente para alcanzar, como, optimizar la productividad y competir en mercados muchos sofisticados. Todo esto indica que el empoderamiento digital hace parte del desarrollo sostenible en el sector empresarial, ya que el dominio en el uso de los recursos tecnológicos por parte de las empresas implica el desarrollo productivo orientado a satisfacer las necesidades actuales de las sociedades con proyecciones a las generaciones venideras, garantizando el equilibrio entre el crecimiento económico, $\mathrm{y}$ el bienestar social.

Asimismo, Gargallo-Castel \& PérezSanz (2009), afirman que el empoderamiento digital implica el dominio en la integración TIC como herramienta de mejora en los procesos y calidad de los servicios, por lo tanto, es imperante que las personas tengan una concepción definida de lo que envuelve a nivel práctico y teórico de lo que significa empoderarse digitalmente. Esto partiendo de la idea que las tecnologías ofrecen diversidad de oportunidades a las organizaciones y la responsabilidad que tienen las universidades para formar ciudadanos capaces de hacer frente a las demandas que los mercados y las 
sociedades exigen, urge la gestión de las mismas (tecnologías información comunicación) como un valor agregado a la formación de los futuros profesionales como mecanismo para la producción y desarrollo empresarial. Asimismo, es importante tener presente que los mercados están ofreciendo herramientas cada vez más sofisticadas que buscan mejorar los servicios.

En este orden de ideas, las universidades como instituciones gestoras del conocimiento y de la formación profesional de ciudadanos, capaces de desenvolverse en mundo globalizado, impregnado de los avaneces tecnológicos y científicos se hace cada vez más competitivo, por lo que el dominio tecnológico se hace impostergable. Las universidades tienen la obligación se transmitir nuevas formas de aprendizaje y nuevas formas para la construcción del conocimiento, que contribuyan al desarrollo económico y al cuidado del ambiente. En este sentido, todo proceso educativo en todos los niveles debe estar direccionado, razón por la cual la capacitación de los docentes es un imperativo e ir implementando nuevos modelos de educación según sus particularidades culturales, económicas, tecnológicas, entre otras. Al mismo tiempo que se gestiones reformas en el currículo que presente el uso de las tecnologías como parte esencial para la formación de los estudiantes y no como una herramienta académica alternativa.

Entonces, resulta importante enfatizar papel que desempeñan los docentes para optimizar sus prácticas educativas y renovar sus estrategias en los procesos de educativos, para lo cual sus estrategias y recursos pedagógicos deberán están basados en la apropiación e integración de las herramientas digitales que ofrecen el uso de las TIC, y en la planificación educativa. En el diseño y producción de TIC para la formación. Al respecto Almenara-Cabero \& Romero-Tena (2010), señala que el manejo y dominio de las tecnologías debe ser una competencia constante en todo profesional docente y para poder integrarlas como parte de los procesos de formación, ya que no se puede formar a una persona en un área determinada y que obtenga conocimientos sobre aquello para lo que fue formado y en la práctica se vea limitado por no saber asirse de las herramientas que los mercados ofrecen.

Atendiendo a este planteamiento la sociedad del conocimiento plantea nuevas metodologías que deroguen las formas tradicionales de educación basadas en métodos tradicionales y aprendizajes memorísticos. En razón de ello, los aprendizajes modernos dinamizan las formas para construir el conocimiento, incluyendo en su reportorio estratégico la interactividad online, conducida por la selección de información apropiada, misma que se procesa para establecer una posición argumentativa de los diferentes puntos de vista transdisciplinario, edificando una red virtual de intercambio continuo entre los usuarios que forman parte de del proceso educativo, en la cual el binomio docente-estudiantes debe estar preparado ante el reto que significa producción de saberes y desarrollo económico sostenible. (Heras-Montoya, Roa-Rivera \& Espinosa-Pulido, 2015).

Según la Unesco (2008), los docentes deben reconocer dónde, cuándo y cómo en los procesos educativos, para ello ameritan estar empoderado digitalmente $y$ tener bien definidas sus funciones como profesional. Es decir, que desarrollen habilidades para su integración didáctica, lo que deriva en el desarrollo de competencias básicas tecnológicas, y esto a su vez en poder de discernimiento entre las metodologías educativas apropiadas, bien sean las que comúnmente emplea rediseñándolas 0 diseñando nuevas formas de enseñanza aprendizaje.

Continuando con la idea central de este estudio Morales-Morales (2016), afirma que el empoderamiento digital es una forma de hacer frente a las realidades sociales y a los avances que la globalización ha impulsado de forma vertiginosa: Toda empresa $u$ organización debe ser competitiva y para ellos 
debe apegarse a los sistemas de producción vanguardistas y modernos. Adaptar su estructura organizativa y procedimental a metodologías computarizadas y programas que faciliten el acceso a la información y derribar las limitaciones de tiempo y espacio. Producir mediante mecanismos que simplifique los esfuerzos y rebasen los límites de expansión productiva. Ahora bien, para establecer una concepción teórica referida al empoderamiento digital es necesario tomar en cuenta algunos elementos claves que lo vincula con los sectores de producción y con el desarrollo sostenible, en virtud de ello se presenta una imagen que resumen las consideraciones teóricas en las cuales se enfoca este estudio, las cuales fueron recogidas e interpretadas de la literatura consultada (ver Figura 1):

\section{Figura 1}

\section{Concepción Teórica Referida al Empoderamiento Digital}

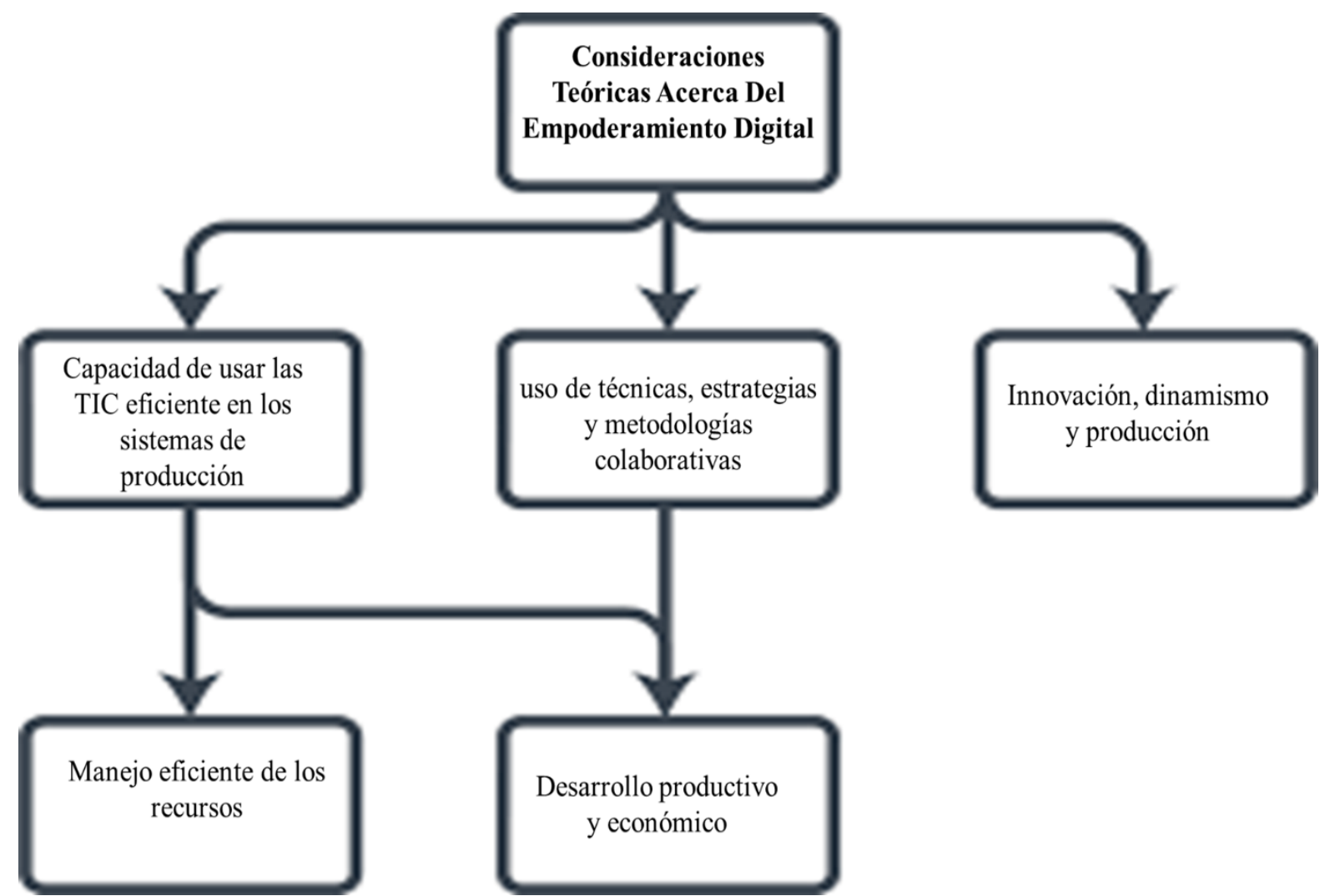

Nota. Elementos claves que lo vincula con los sectores de producción y con el desarrollo sostenible, elaboración propia (2021).

\section{Implicaciones del Empoderamiento Digital}

Cuando de empoderamiento digital se trata las personas adquieren una forma de pensamiento más abierto, flexible, crítico y se hacen conscientes de sus responsables, personales y sociales al momento de hacer usos de la red. Asimismo, aprende a buscar, clasificar, seleccionar la información según las necesidades y los objetivos que se ha planteado. De igual manera, su utilización las convierte en medios de comunicación: personal, institucional y de difusión masiva, si así lo requiere la empresa. Todo esto repercute en las formas de dar respuesta a las necesidades que se presenten dentro de una organización (Guitert, 2013).

Entonces, la integración de las TIC conjuntamente con el potencial y dominio de los usuarios para emplearlas en su campo laboral permite crear nuevos conductos y 
procedimientos de producción, ya que dejan ver nuevas formas de intervención ante las necesidades sociales, en este caso, de Colombia, es decir, contribuye con desarrollo sostenible del sector empresarial. Por otra parte, facilita el intercambio de información, lo que representa un punto de anclaje para la planificación y proyecciones en relación con el rumbo en el cual se deben direccionar las empresas y poder satisfacer las demandas de consumo y de servicio en Colombia.

Finalmente, el Plan Nacional de TIC (2008-2019) señala que el Gobierno Nacional se ha comprometido para que todos los colombianos se informen y se comuniquen haciendo uso eficiente y productivo de las TIC, para mejorar la inclusión social y aumentar la competitividad. Asimismo, puntualiza que en Colombia, el sector de desarrollo de software está orientado a la comercialización de productos hacia lo interno del país, el cual tiene bajos volúmenes por su poco desarrollo, sin trayectoria como exportador ( Cruz-Rodríguez, 2019).

Lo que indica una fuerte voluntad técnica y política en la instrumentación de políticas públicas orientadas al fortalecimiento la capacidad tecnológica, para el uso de las TIC en el sector de empresarial sin importar su naturaleza u objeto social. Por todo lo anterior, existe una alta apropiación de tecnologías de información y comunicación en empresas grandes, las cuales representan solo el $14 \%$ del parque empresarial. En ese contexto hay evidencias de rezago digital en empresas medianas con experticia exportadora en el área agrícola, pero muy tradicional en el manejo de las TIC y por ende rezagada en su condición de empresa con empoderamiento digital. Según los documentos consultados, tales como el Diagnostico del Ministerio de Comunicaciones hay sectores empresariales con importantes avances en la introducción de las TIC, que mejora su sector, pero con poca influencia sobre el empoderamiento digital del ciudadano usuario de esos servicios. Por último, se observa un mayor uso de las TIC en empresas grandes y un uso más limitado en las empresas pequeñas.

Partiendo de los valores obtenidos de la aplicación de los instrumentos de investigación, a los cuales se les hace el análisis y la discusión de los resultados obtenidos en el proceso de recolección de la información. Los datos (ver Tabla 1) se presentan siguiendo el orden de aparición de cada uno de los indicadores y dimensiones de las variables.

Tabla 1

Datos Generales de la Dimensión Concepción Teórica Acerca del Empoderamiento Digital

\begin{tabular}{|c|c|c|c|c|c|c|c|c|c|c|}
\hline $\begin{array}{l}\text { Indicador } \\
\text { Alt. De } \\
\text { Respuesta } \\
\end{array}$ & Siempre & & \multicolumn{2}{|c|}{$\begin{array}{l}\text { Casi } \\
\text { Siempre }\end{array}$} & \multicolumn{2}{|c|}{ Algunas Veces } & \multicolumn{2}{|l|}{$\begin{array}{l}\text { Casi } \\
\text { Nunca }\end{array}$} & \multicolumn{2}{|c|}{ Nunca } \\
\hline Población & Doc. & $\begin{array}{l}\text { Est. } \\
\text { Trab. }\end{array}$ & Doc. & $\begin{array}{l}\text { Est. } \\
\text { Trab. }\end{array}$ & Doc. & $\begin{array}{l}\text { Est. } \\
\text { Trab. }\end{array}$ & Doc. & $\begin{array}{l}\text { Est. } \\
\text { Trab. }\end{array}$ & Doc. & $\begin{array}{l}\text { Est. } \\
\text { Trab. }\end{array}$ \\
\hline Porcentaje & $\%$ & $\%$ & $\%$ & $\%$ & $\%$ & $\%$ & $\%$ & $\%$ & $\%$ & $\%$ \\
\hline $\begin{array}{l}\text { Dominio en la } \\
\text { integración } \\
\text { TIC }\end{array}$ & 0,00 & 11,32 & 0,00 & 7,55 & 0,00 & 24,53 & 44,44 & 56,60 & 55,56 & 0,00 \\
\hline $\begin{array}{l}\text { Concepción } \\
\text { del término } \\
\text { empoderamie } \\
\text { nto digital }\end{array}$ & 0,00 & 9,43 & 0,00 & 47,17 & 0,00 & 13,2 & 44,44 & 30,2 & 55,56 & 0,0 \\
\hline $\begin{array}{l}\text { Procesos } \\
\text { innovadores }\end{array}$ & 0,00 & 9,43 & 0,00 & 32,08 & 0,00 & 30,19 & 11,11 & 28,30 & 88,89 & 0,0 \\
\hline Promedio & 0,00 & 10,06 & 0,00 & 28,93 & 0,00 & 22,64 & 33,33 & 38,36 & 66,67 & 0,00 \\
\hline Porcentaje & 5,03 & & 14,47 & & 11,32 & & 35,85 & & 33,33 & \\
\hline
\end{tabular}

Nota. Valores obtenidos de la aplicación de los instrumentos de investigación, elaboración propia (2021). 
Analizada la Tabla 1 se buscó establecer la concepción teórica acerca del empoderamiento digital, en relación a ello se realizaron una serie de interrogantes a docentes universitarios y estudiantes que han incursionando en sector productivo laboral, observándose que un $35,85 \%$ de encuestados opinaron que casi nunca reconocen la concepción teórica del término empoderamiento, realizan procesos de innovación, participan en programas de capacitación tecnológica, han participado en foros digitales, utilizan recursos tecnológicos en clase. Asimismo, el 33,33\% manifestó que nunca participan de esos elementos, el $14,47 \%$ señalo casi siempre hacerlo, el $11,32 \%$ expresa realizarla algunas veces y el $5,03 \%$ siempre lo hacen. Igualmente se observa tomando en cuenta el promedio de directivos $33,33 \%$ que se presentan una enfatizada debilidad con respecto a los indicadores dominio en la integración TIC, concepción del término empoderamiento digital y procesos innovadores con un $44,44 \%$, en cuanto a la opinión de los docentes en referencia a su promedio de respuesta $38,36 \%$ se observan igualmente debilidades.

En cuanto a las debilidades o dificultades que se presentan en los indicadores se puede resaltar que por parte de los directivos y docentes del indicador
Procesos innovadores se presenta como una dificultad con un $11,11 \%$ en opinión de los estudiantes y en cuanto a los docentes con un $28,30 \%$ respectivamente, evidenciándose también para la población docente dificultad en la identificación sobre la concepción del término empoderamiento digital, con un $30,02 \%$. Concluyendo este análisis se puede resaltar que se presenta la mayor preferencia de respuestas por parte de los directivos y docentes en las alternativas casi nunca y nunca lo que significa que la dimensión concepción teórica acerca del empoderamiento digital, representando una concepción teórica deficiente dentro de la variable objeto de estudio.

En la Tabla 2 se puede observar que según la opinión emitida por los docentes y estudiantes encuestados. Casi Siempre el desarrollo sostenible va en función de la productividad y del grado de conocimiento para integrar las herramientas que los mercados ofrecen, el desarrollo económico y la calidad de servicios con un $33,26 \%$, seguida de Siempre con un 29,63\%, posteriormente la opción Casi Nunca con un $24,21 \%$, seguido por un $8,49 \%$ que Casi Nunca las distingue los elementos del desarrollo sostenible y finalmente un $4,40 \%$ de la población que lo hace algunas veces.

Tabla 2

Datos Generales Desarrollo Sostenible del Sector Empresarial

\begin{tabular}{|c|c|c|c|c|c|c|c|c|c|c|}
\hline \multirow{2}{*}{$\begin{array}{l}\text { Indicador } \\
\text { Alt. De } \\
\text { Respuesta } \\
\text { Población }\end{array}$} & \multicolumn{2}{|c|}{ SIEMPRE } & \multicolumn{2}{|c|}{$\begin{array}{l}\text { CASI } \\
\text { SIEMPRE }\end{array}$} & \multicolumn{2}{|c|}{$\begin{array}{l}\text { ALGUNAS } \\
\text { VECES }\end{array}$} & \multicolumn{2}{|c|}{$\begin{array}{l}\text { CASI } \\
\text { NUNCA }\end{array}$} & \multicolumn{2}{|c|}{ NUNCA } \\
\hline & Doc. & $\begin{array}{l}\text { Est. } \\
\text { Trab. }\end{array}$ & Doc. & $\begin{array}{l}\text { Est. } \\
\text { Trab. }\end{array}$ & Doc. & $\begin{array}{l}\text { Est. } \\
\text { Trab. }\end{array}$ & Doc. & $\begin{array}{l}\text { Est. } \\
\text { Trab. }\end{array}$ & Doc. & $\begin{array}{l}\text { Est. } \\
\text { Trab. }\end{array}$ \\
\hline Porcentaje & $\%$ & $\%$ & $\%$ & $\%$ & $\%$ & $\%$ & $\%$ & $\%$ & $\%$ & $\%$ \\
\hline Producción & 44,44 & 0,00 & 55,56 & 32,08 & 0,00 & 7,55 & 0,00 & 43,40 & 0,00 & 16,98 \\
\hline Desarrollo económico & 66,67 & 0,00 & 33,33 & 20,8 & 0,00 & 13,21 & 0,00 & 47,17 & 0,00 & 18,87 \\
\hline Calidad de servicios & 66,67 & 0,00 & 33,33 & 24,53 & 0,00 & 5,66 & 0,00 & 54,72 & 0,00 & 15,09 \\
\hline Promedio & 59,26 & 0,00 & 40,74 & 25,79 & 0,00 & 8,81 & 0,00 & 48,43 & 0,00 & 16,98 \\
\hline Porcentaje & 29,63 & & 33,26 & & 4,40 & & 24,21 & & 8,49 & \\
\hline
\end{tabular}

Nota. Opinión emitida por los docentes y estudiantes encuestados, elaboración propia (2021).

Se puede evidenciar a través del promedio obtenido para los docentes y estudiantes de 
40,74\% la fortaleza para el indicador Producción con un 55,56\% y para los estudiantes bajo su promedio de $25,79 \%$ se aprecian fortalezas en el mismo indicador con un 32,08\%. Apreciando debilidad o limitaciones en cuanto a los indicadores Desarrollo económico y calidad de servicios para los docentes con un $33,33 \%$, y en el caso de los estudiantes se evidencia al indicador calidad de servicios con un 24,53\% y al indicador desarrollo económico con un 20,8\%. Al analizar la inclinación que tiene la población encuestada según la categoría de respuesta se puede evidenciar que existe una inclinación hacia las categorías Casi Siempre y Siempre lo que indica que se cumple de manera adecuada con la distinción de los indicadores de la dimensión.

Alcanzados los objetivos con carácter descriptivo, diseñados para dar fortaleza a esta investigación, corresponde entonces, la aplicación de un estadístico que permita inferir estos valores o resultados hacia la población, por lo tanto, se decidió la aplicación del método del cálculo del
Coeficiente de Correlación de Spearman, para Establecer el grado de relación entre la concepción teórica del empoderamiento digital y el desarrollo sostenible empresarial. Para lo cual se transformaron las mediciones a forma nominal al compararlas con el baremo, utilizando los valores recogidos en las matrices de doble entrada, anexas, con el auxilio del programa SPSS foro Windows v.21.0:

El procedimiento utilizado para la prueba fue a través de la fórmula estadística siguiente y corroborada por los resultados obtenidos de la aplicación del programa estadístico SPSS v. 21.0

$$
\rho=1-\frac{6 \sum d^{2}}{n(n+1)(n-1)}
$$

Donde:

$\rho$ : Coeficiente de correlación de Spearman

d: diferencia entre los rangos $(\mathrm{X}-\mathrm{Y})$

n: número de datos

Tabla 3

Correlación entre las Variables Concepción Teórica del Empoderamiento Digital y el Desarrollo Sostenible Empresarial

\begin{tabular}{|c|c|c|c|c|}
\hline & & & $\begin{array}{l}\text { Concepción } \\
\text { teórica del } \\
\text { empoderamiento } \\
\text { digital }\end{array}$ & $\begin{array}{l}\text { Desarrollo } \\
\text { sostenible } \\
\text { empresarial }\end{array}$ \\
\hline \multirow[t]{6}{*}{$\begin{array}{l}\text { Rho de } \\
\text { Spearman }\end{array}$} & \multirow{3}{*}{$\begin{array}{l}\text { Concepción } \\
\text { teórica del } \\
\text { empoderamiento } \\
\text { digital }\end{array}$} & $\begin{array}{l}\text { Coeficiente } \\
\text { correlación }\end{array}$ & 1,000 &, $763^{*}$ \\
\hline & & Sig. (bilateral) & . & 033 \\
\hline & & $\mathrm{N}$ & 56 & 56 \\
\hline & \multirow{3}{*}{$\begin{array}{l}\text { Desarrollo } \\
\text { sostenible } \\
\text { empresarial }\end{array}$} & $\begin{array}{l}\text { Coeficiente } \\
\text { correlación }\end{array}$ &, $763^{*}$ & 1,000 \\
\hline & & Sig. (bilateral) &, 033 & \\
\hline & & $\mathrm{N}$ & 56 & 56 \\
\hline
\end{tabular}

Nota. *La correlación es significativa al nivel 0,05 (bilateral), elaboración propia (2021).

Aplicada la fórmula se obtuvo un coeficiente de correlación de Spearman 0,763 de a un nivel de significancia de 0,033 , ello indica que hay una relación alta y estadísticamente significativa entre las variables, su signo positivo indica con ello que a medida que aumentan los valores de la variable concepción teórica del empoderamiento digital aumenta de forma alta el Desarrollo sostenible empresarial y viceversa.

\section{Discusión}

Después de tabular los resultados y descritos estadísticamente con base a las interrogantes formuladas a los encuestados se tiene que respecto a la variable concepción teórica acerca del empoderamiento digital, se observa una debilidad significativa, respecto 
a la concepción que se tiene respecto de la misma. Lo que indica la integración de las TIC a los procesos productivos son limitadas, debido al poco conocimiento que tienen los usuarios para su debido y potencial manejo. Resultados que discrepan de la teoría de Gargallo \& Pérez (2009), quienes afirman que el empoderamiento digital implica el Dominio en la integración TIC como herramienta de mejora en los procesos y calidad de los servicios, por lo tanto, es imperante que las personas tengan una concepción definida de lo que envuelve a nivel práctico y teórico de lo que significa empoderarse digitalmente, como parte de los procesos innovadores.

Seguidamente, se observa que en cuanto a la variable desarrollo sostenible del sector empresarial, los resultados muestran concordancia con la teoría de Jiménez-Pitre (2017), ya que ellos exponen la necesidad de producción intelectual, es decir que el conocimiento o empoderamiento digital es el fundamento para la producción de bienes y servicios en cualquier ramo del quehacer de las personas en la actualidad. Por lo que urgen esta competencia como parte del desarrollo de las naciones, razón por la cual las universidades deben formar parte de la formación para su posterior incorporación al aparato productivo colombianos en su plenitud, ya que esto forma parte del desarrollo económico y la calidad de los servicios.

\section{Conclusiones}

Respecto a los objetivos del estudio se deduce con base a los resultados del mismo que el empoderamiento digital es el resultado de un grado elevado de conocimiento en el manejo de las TIC, además, la concepción teórica parte lo planteado en el Plan Nacional de TIC (2008-2019), y Cruz-Rodríguez (2019); en Colombia se ha realizado esfuerzos, tanto en el sector público como en el privado, para promover la utilización TIC en las empresas.

Estos esfuerzos han sido poco coordinados entre ambos sectores, pero han coincidido con una serie de eventos muy favorables por la existencia de inversión en ambos sectores. No obstante, pese a los esfuerzos realizados aún persisten brechas para su integración, mismas que obedecen al poco empoderamiento de los usuarios o trabajadores para su adecuada implementación. Sin embargo, se ha logrado incorporar con éxito en algunas empresas ligados a las áreas bancarias, turísticohotelero y otros grupos de empresas de mayor tamaño. Sin embargo, otras empresas de menor tamaño y del área de manufactura tradicional se encuentran rezagadas, debido a falta de dominios para hacer uso eficiente de las TIC.

\section{Referencias}

Almenara-Cabero, J., \& Romero-Tena, R. (2010). Diseño y producción de TIC para la formación. Editorial UOC.

Carruyo, N., Ureña, Y.C. \& Quiñones, E. (2017). Digital empowerment: integration university, business and public policy for endogenous development. 16.

Cruz-Rodríguez, E. C. (2019). Importancia del manejo de competencias tecnológicas en las prácticas docentes de la Universidad Nacional Experimental de la Seguridad (UNES). Revista Educación 43(1). https://www.redalyc.org/journal/440/44057415013/ $\mathrm{html} /$.

Gargallo-Castel, A., \& Pérez-Sanz, J. (2009). El papel de las tecnologías de la información y la comunicación en las empresas de economía social, 28.

Garza-Mercado, A. (2007). Manual de técnicas de investigación para estudiantes de ciencias sociales y humanidades. Séptima edición. El colegio de México.

https://books.google.co.ve/books?id=jdaQtk8RK2s $\mathrm{C} \& p g=\mathrm{PA} 18 \& \mathrm{dq}=$ investigacion + analitica\&hl=es419\&sa $=$ X\&ved $=2$ ahUKEwjUiJiKz5zzAhWBlWoF HTGEAkgQ6AF6BAgIEAI\# $\mathrm{v}=$ onepage \&q=investi gacion $\% 20$ analitica $\& \mathrm{f}=$ false

Heras-Montoya, V., Roa-Rivera, R.I \& Espinosa-Pulido, A. (2015). Las competencias digitales de futuros docentes y su relación con los estándares internacionales en TIC. Revista Iberoamericana de Producción Académica y Gestión Educativa. https://www.pag.org.mx/index.php/PAG/article/vie $\mathrm{w} / 275$

Jiménez-Pitre, I.A, Martelo-Gómez, R.J. Chiquillo-Rodelo, J. Lloreda-Gracia, D.J. \& Morales-Camacho, M.S. (2017). Estrategias para el empoderamiento digital y la integración de universidades con sectores clave para la sociedad colombiana. Revista Lasallista de investigación https://doi.org/10.22507/rli.v14n1a10 14(1),112-25. 


\section{Consideraciones Teóricas Acerca del Empoderamiento Digital y Desarrollo Sostenible del Sector Empresarial en Colombia.}

Méndez, A. \& Astudillo, M. (2008). La investigación en la era de la información. Guía para realizar la bibliografia $y$ fichas de trabajo. http://www.economia.unam.mx/academia/inae/pdf/i nae1/u115.pdf

Morales-Morales, E. (2016). Empoderamiento y transformación de las relaciones de poder. Un análisis crítico de los procesos institucionales de participación ciudadana. https://www.tdx.cat/bitstream/handle/10803/400078 /emm1de1.pdf?sequence $=1$

Mochi, P. (2012). Programas para la inclusión digital y la concertación de actores en procesos de desarrollo territorial, 37.

Rodríguez-Pérez, M. A.; Acuña-Martínez, J. A., Rojas-Vera, L. R., Lobato, L. A. (2015). Linking State, University And Business In Nicaragua. journal of Human Sciences, 21.

Plan Nacional de Tecnologías de la Información y las Comunicaciones (2008). Todos los colombianos conectados, todos los colombianos informados. https://eduteka.icesi.edu.co/pdfdir/ColombiaPlanNa cionalTIC.pdf

Tamayo y Tamayo, M. (2009). El proceso de la investigación científica incluye evaluación $y$ administración de proyectos de investigación. Limusa.

Unesco (2008). Estándares de Competencias en TIC para Docentes

http://www.unesco.org/new/fileadmin/MULTIMED IA/FIELD/Santiago/pdf/Competencias-estandaresTIC.pdf 